\title{
Review of light flavour physics on the lattice
}

\author{
Andreas Jüttner* \\ School of Physics and Astronomy \\ University of Southampton \\ Highfield \\ Southampton, SO17 1AJ, UK \\ E-mail: juettner@soton.ac.uk
}

For quite some time now simulations of lattice QCD have allowed for predicting a basic set of light flavour quantities reliably and with increasingly high precision. The field has started to move on: Advances in field theory, algorithms and computing for the first time allow to address more complicated problems like the conceptually clean inclusion of electromagnetic and isospin effects for hadronic matrix elements, rare kaon decays or $\varepsilon_{K}$ and the $K_{L}-K_{S}$ mass-difference. This talk aims at providing an overview over the state-of-the-art and beyond.

The 33rd International Symposium on Lattice Field Theory

14 -18 July 2015

Kobe International Conference Center, Kobe, Japan

${ }^{*}$ Speaker. 


\section{Introduction}

This is the write-up of a talk in which I reported on the status of lattice QCD simulations for mesons containing the light valence quarks $u, d$ and $s$, focusing on flavour phenomenology. The talk included a critical assessment of recent results and an outlook. But given the high level of activity in the field this talk could highlight only a small set of recent efforts. For a more comprehensive overview in particular of results readily applicable to wider Standard Model (SM) phenomenology the reader should consult the reports prepared by Flavour Lattice Averaging Group (FLAG) $[1,2,3]$.

The topics covered in this talk split into two classes:

Standard quantities: computations where systematic errors are under good control and the main objective is increased precision in view of applications to tests of the SM. These quantities are covered in detail also in the FLAG-report [1,2].

Beyond standard quantities: computations which require to go beyond known and established lattice techniques (theory and algorithms). Rather than about precision it is about being able to do the computation at all and to identify and understand sources of systematic uncertainties.

Section 2 summarises state-of-the-art results for the pion and kaon decay constants and the $K \rightarrow \pi$ semileptonic form factor and neutral kaon mixing which by now are standard quantities. This section also contains some critical remarks on how the progress in simulation quality, in particular the availability of ensembles with physical light sea and valence quarks, allows to remove hitherto dominant sources of systematics in the data analysis. Section 3 provides an overview over efforts to inlcude QED effects in calculations of hadronic matrix elements and the computation of long distance effects in neutral kaon mixing and rare kaon decays. Section 4 discusses results following a new idea for predicting hadronic transition matrix elements in the timelike regime.

\section{Standard quantities}

For quite some time now lattice QCD predictions for simple meson matrix elements (with at most one meson in the initial/final state) do play a decisive role in flavour phenomenology. Experimental measurements of leptonic or semileptonic meson decays and neutral meson mixing are only useful for SM phenomenology if the relevant hadronic matrix elements are known reliably and precisely. This section provides a brief overview.

\subsection{Leptonic and semileptonic tree-decays}

In 2004 Marciano [4] suggested to use the lattice determination of $f_{K} / f_{\pi}$ to determine $\left|V_{u s} / V_{u d}\right|$ using the relation

$$
\frac{\Gamma\left(K \rightarrow \mu \bar{v}_{\mu}(\gamma)\right)}{\Gamma\left(\pi \rightarrow \mu \bar{v}_{\mu}(\gamma)\right)}=\frac{\left|V_{u s}\right|^{2}}{\left|V_{u d}\right|^{2}}\left(\frac{f_{K^{ \pm}}}{f_{\pi^{ \pm}}}\right)^{2} \frac{m_{K}\left(1-m_{\mu}^{2} / m_{K}^{2}\right)^{2}}{m_{\pi}\left(1-m_{\mu}^{2} / m_{\pi}^{2}\right)^{2}} \underbrace{\left(1+\delta_{\mathrm{EM}}^{\mathrm{ChPT}}\right)}_{0.9930(35)} .
$$

The analysis of available experimental data [5] leads to $\left|V_{u s} / V_{u d}\right| f_{K^{+}} / f_{\pi^{+}}=0.2758(5)$. Note that in this equation the decay constants are the ones with $m_{u}$ and $m_{d}$ assuming their physical value ( $S U(2)$ isospin broken) but the electromagnetic coupling set to zero, $\alpha_{\mathrm{EM}}=0$. The QED corrections (indicated with the underbrace in eq. (2.1)) have been applied based on estimates in chiral 
perturbation theory. In chiral perturbation theory the $S U(2)$ breaking effect is expected to be of the same magnitude as the QED correction [6, 7] underlining that both corrections need to be taken into account. A recent update by Moulson provides a slightly more precise number 0.2760(4) [8]. The improved error is mainly down to improved experimental analyses of branching ratios.

The SM prediction for semileptonic decays is

$$
\Gamma(K \rightarrow \pi l v)=C_{K}^{2} \frac{G_{F}^{2} m_{K}^{5}}{192 \pi^{3}} S_{\mathrm{EW}}\left(1+\delta_{S U(2)}^{\mathrm{ChPT}}+\delta_{\mathrm{EM}}^{\mathrm{ChPT}}\right)^{2} I\left(f_{+}^{K^{0} \pi^{-}}(0)\left|V_{u s}\right|\right)^{2} .
$$

$K^{0} \rightarrow \pi^{-}$is the reference channel which the lattice is expected to predict and the Clebsh-Gordon $C_{K}$ and the isospin and electromagnetic corrections $\delta_{S U(2)}$ and $\delta_{\mathrm{EM}}$, respectively, are adjusted to allow for a combined analysis of various measured experimental channels $\left(K_{L e 3}, K_{L \mu 3}, K_{S e 3}, K_{e 3}^{ \pm}\right.$ and $\left.K_{\mu 3}^{ \pm}\right)$. The form factor $f_{+}(0)$ is defined through the QCD matrix element

$$
\left\langle\pi\left(p_{\pi}\right)\left|V_{\mu}(0)\right| K\left(p_{K}\right)\right\rangle=f_{+}^{K \pi}\left(q^{2}\right)\left(p_{K}+p_{\pi}\right)_{\mu}+f_{-}^{K \pi}\left(q^{2}\right)\left(p_{K}-p_{\pi}\right)_{\mu} .
$$

and in most phenomenological analyses only its value from the lattice at zero momentum transfer $q^{2}=0$ enters and it's shape (entering $I$ ) is taken from experiment. Based on the analysis in [5] the experimental status can be summarised by $\left|V_{u s}\right| f_{+}^{K^{0} \rightarrow \pi^{-}}(0)=0.2163(5)$ where the electromagnetic and strong isospin corrections are based on estimates in chiral perturbation theory [9, 10]. A recent update by Moulson has a slightly reduced error, $0.2165(4)[11,8]$. The reduction in error is mainly down to improved estimates of strong isospin breaking effects (quark mass ratios) based on FLAG [2].
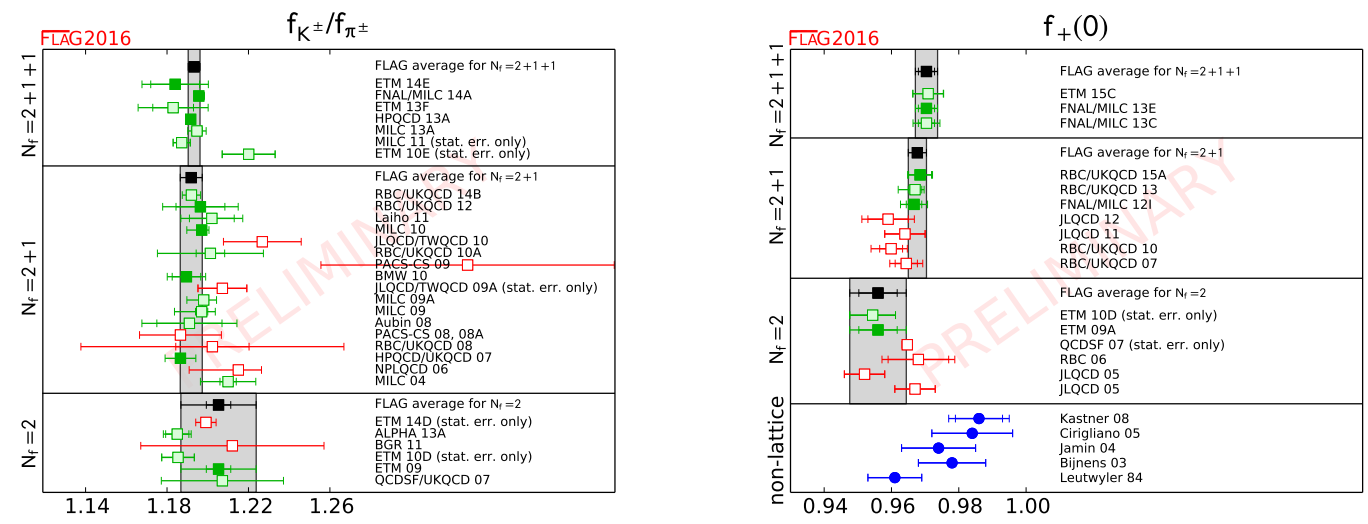

Figure 1: Preliminary FLAG plots for the ratio of kaon and pion decay constants and the semileptonic vector form factor at zero momentum transfer.

Figure 1 shows the preliminary update of the world data on $f_{K^{+}} / f_{\pi^{+}}$and $f_{+}^{K^{-} \rightarrow \pi^{0}}(0)$ from the upcoming 2016 FLAG review [3]. The precision with which these quantities can now be predicted is below $0.3 \%$ and this has become possible only recently through the availability of gauge ensembles with very close-to-physical dynamical light quark masses in large volume. Note that before all results were based on simulations with unphysically heavy dynamical and valence light quark masses which had to be extrapolated to the physical quark masses guided by chiral perturbation 
theory or by models. With the increased availability of gauge ensembles with (close to) physical light quark masses the residual correction towards the exact physical quark mass no longer dominates the error budget. Instead the statistical error, cutoff effects and finite volume errors now are the dominant sources of uncertainties.

Figure 2 shows FLAG's preliminary update of 1st-row CKM unitarity for $N_{f}=2,2+1,2+$ $1+1$ flavour results. Despite the progress in the quality of results from the lattice no qualitatively new picture has arisen and 1st row unitarity is confirmed.

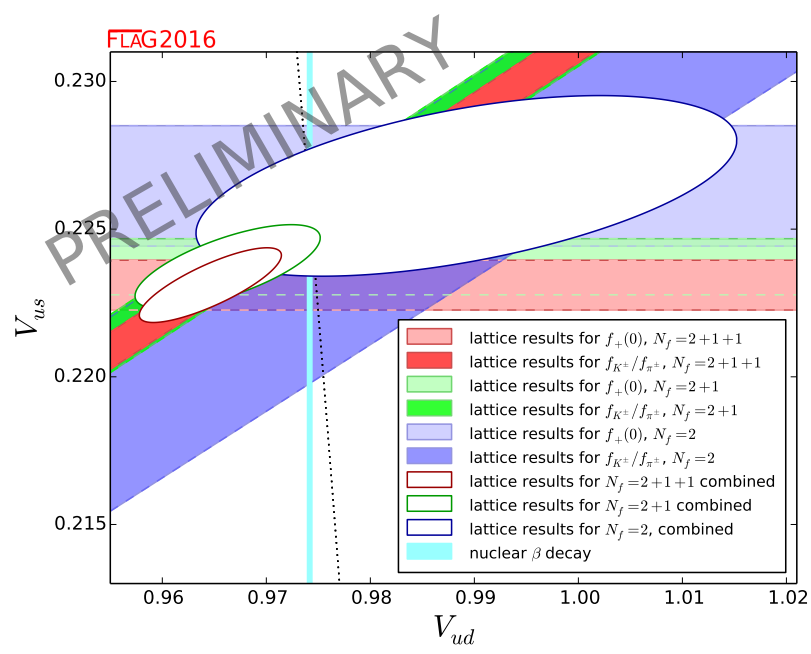

Figure 2: FLAG's illustration of the first-row CKM unitarity test. The dashed black line corresponds to unitarity $\left|V_{u d}\right|^{2}+\left|V_{u s}\right|^{2}+\left|V_{u b}\right|^{2}=1$. The tilted bands represent the $N_{f}=2,2+1,2+1+1$ results for $f_{K^{+}} / f_{\pi^{+}}$ converted to $\left|V_{u s} / V_{u d}\right|$, and the horizontal bands correspond to the results for $f_{+}^{K^{0} \rightarrow \pi^{-}}(0)$ converted to $\left|V_{u s}\right|$.

The plot on $f_{+}^{K^{-} \rightarrow \pi^{0}}(0)$ in figure 1 includes a recently published data analysis update by the $\mathrm{RBC} / \mathrm{UKQCD}$ collaboration [12]. The novelty in this paper is the inclusion of RBC/UKQCD's physical light quark mass ensembles at two lattice spacings. The study contains a detailed analysis of excited state contributions to the ratio of three point functions by generating results for all possible source-sink separations between the initial and final state pions and kaons. Their results for the vector form factor obtained from the $3 \mathrm{pt}$ function of the vector current as well as the one obtained via the Ward Identity from the scalar current [13] (note that $f_{0}(0)=f_{+}(0)$ ),

$$
\left.\left\langle\pi\left(p_{\pi}\right)|S| K\left(p_{K}\right)\right\rangle\right|_{q^{2}=0}=f_{0}^{K \pi}(0) \frac{m_{K}^{2}-m_{\pi}^{2}}{m_{s}-m_{u}},
$$

are illustrated in figure 3. The observed dependence on the source-sink separation differs between time- and spacial components of the vector current and scalar current, respectively (it is found smaller in magnitude for the zero-component of the vector current compared to the spatial components and the scalar current $3 \mathrm{pt}$ functions). This study underlines the importance of understanding the contributions of excited states in detail. The results for the form factor $f_{+}^{K \rightarrow \pi}(0)$ for the various pion and kaon masses generated by RBC/UKQCD on their set of ensembles with $a=0.11 \mathrm{fm}$ are illustrated in the plots in figure 4. The 1.h.s. plot shows that the data cannot be reconciled 

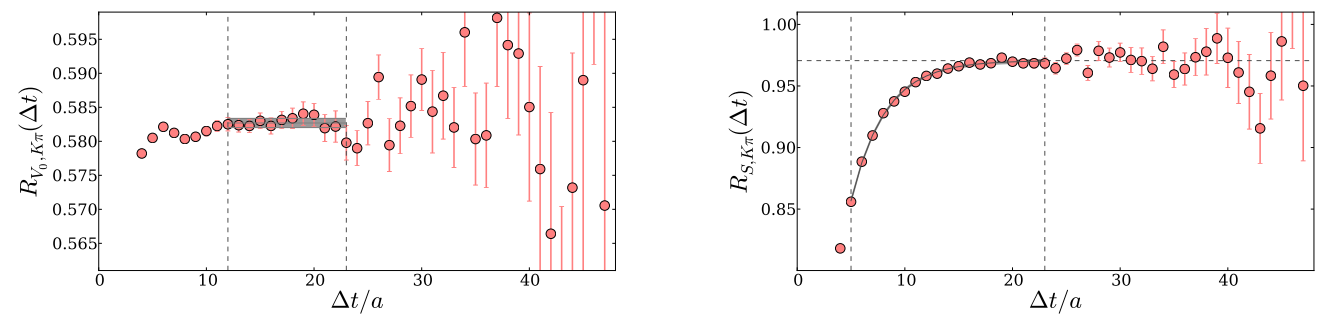

Figure 3: Dependence of the ratio of 3pt functions used by RBC/UKQCD [12] to determine the $K \rightarrow \pi$ vector form factor on the separation of the kaon source and pion sink.

with the NLO prediction of chiral perturbation theory [10] where the only free parameter is the pseudoscalar meson decay constant [14]. Instead, the data suggests a simple linear interpolation in $\left(m_{K}^{2}-m_{\pi}^{2}\right)^{2} / m_{K}^{2}$ as illustrated in the r.h.s. plot. RBC/UKQCD uses this parametrisation, applied at both of their lattice spacings ( $a=0.09,0.11 \mathrm{fm}$ ), respectively, for the interpolation to the exact physical quark mass point. For their final result they only include data from the light quark mass
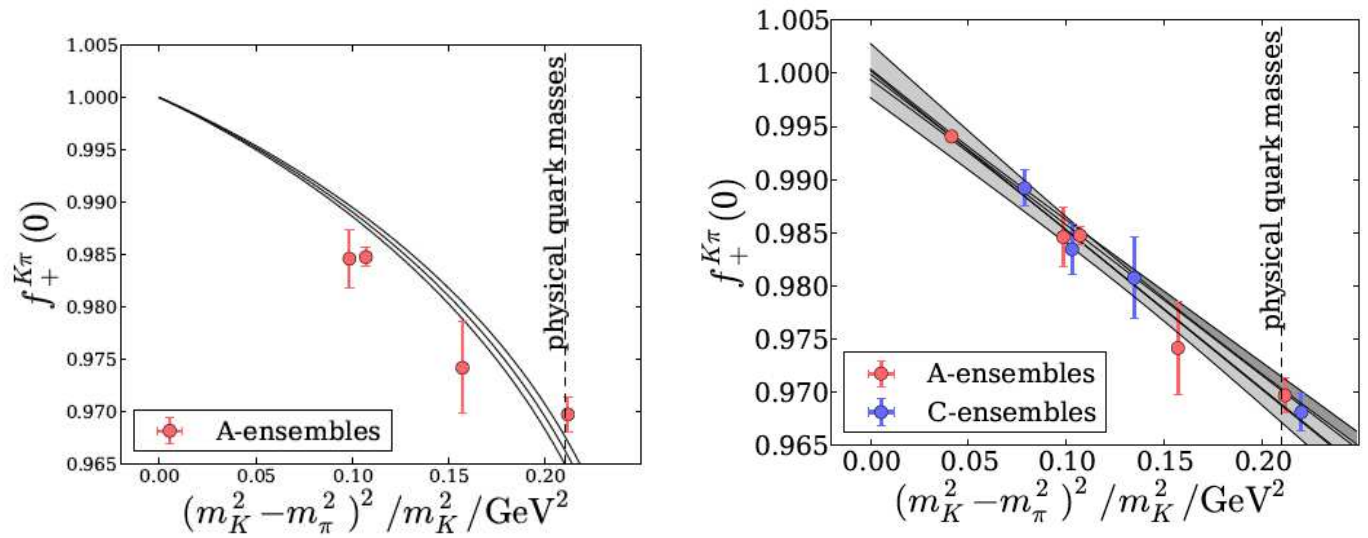

Figure 4: Left: unsuccessful attempt to fit RBC/UKQCD's data for the $K \rightarrow \pi$ form factor with NLO chiral perturbation theory. Right: simple (successful) linear parametrisation of data.

ensembles to guide the interpolation. In this way the interpolated results end up having the same statistical error as the ones on the (near) physical quark mass ensemble with entirely negligible dependence on the model used for the interpolation.

\subsection{Change of the analysis paradigm?}

Here I would like to discuss briefly how the increased availability of ensembles with (near) physical light quark masses is changing the way in which lattice data is analysed. With (near) physical I mean that increasingly the generation of gauge ensembles is done with light as well as strange (and charm) quark masses tuned to their physical values. That is, a pion decay constant evaluated on such ensembles in principle no longer needs to be chirally extrapolated. In practice however there usually remains a slight mistuning which has to be removed by means of a small inter-/extra-polation towards the exact physical point. Reading the recent literature one can identify two different strategies to do so: 
- do a global analysis over a set of ensembles with physical as well as unphysical light quark mass (also include results from different physical volumes and lattice spacings).

This strategy seems preferable in cases where the global fit model is known to be correct (or correct to within the precision goal). The global analysis together with the fit ansatz is known to help reducing the statistical error on the final result (often because of their cheaper simulation cost, results at unphysically heavy quark mass parameters have smaller statistical error and hence can better constrain the fit)

- physical point analysis - use the result at unphysical quark masses merely to correct for small mistunings in the quark mass. This analysis strategy depends less on the particular form of the fit ansatz and is the ansatz of choice where model-independence is the paradigm.

Let me illustrate the two strategies based on three recent results:

RBC/UKQCD's overweighting [15]: The fit ansatz is based on heavy meson ChPT [16, 17] or polynomial ansätze for the quark mass dependence. RBC/UKQCD carry out a global fit over their comprehensive set of gauge ensembles giving the (near) physical point ensembles (of which they have two at $1 / a=1.8,2.4 \mathrm{GeV}$, respectively) a particularly large weight factor $\omega_{i}$ in the $\chi^{2}$ function (otherwise $\omega_{i}=1$ ),

$$
\chi^{2}=\sum_{i} \frac{\omega_{i}\left[y_{i}-f\left(x_{i}, \vec{a}\right)\right]^{2}}{\sigma_{i}^{2}} .
$$

The large weight is chosen such that the fit curve will go exactly through the data point with the (near) physical $m_{l}$. Given that these data are afflicted by only a small mistuning with respect to the actual physical quark mass the result after the correction turns out to carry the same statistical error. In this way the results for unphysically heavy quark masses merely guide the inter-/extrapolation but they are not used to reduce the statistical error of the final result. In RBC/UKQCD's analysis the final error budget is completely dominated by the statistical error.

HPQCD's global analysis [18]: A global fit ansatz over ensembles with $m_{\pi} \approx m_{\pi}^{\text {phys. }}, \ldots, 300 \mathrm{MeV}$ is used. It is based on NLO partially quenched Chiral perturbation theory with models for higher order terms, cutoff effects and finite volume effects. This fit does not give any particular weight to the results with (near) physical quark masses and profits from the small statistical error on all ensembles towards a particularly precise result after the extrapolation.

FNAL/MILC's physical mass analysis [19]: On their (near) physical quark mass ensembles this collaboration generates a number of partially quenched (PQ) quark mass points in the vicinity of their expected physical values. They then determine the lattice spacing, the physical light and strange quark mass individually on each ensemble. This is achieved by interpolating the PQ results for $m_{\pi}^{2} / f_{\pi}^{2}$ towards their physical value [20]. This allows them to determine the lattice scale by using the physical value of the pion decay constant as input. A similar analysis follows for the strange and charm quark mass. The small interpolations for the light quarks are based on NLO partially quenched chiral perturbation theory. Their analysis strategy reduces the dependence on unphysically heavy simulation points and the fit model to a minimum.

This new generation of simulations achieves unprecedented precision. With the error budget being reshuffled due to the absence of long chiral extrapolations, other uncertainties have to be controlled with much better care. An example is illustrated in figure 5. The two plots show 

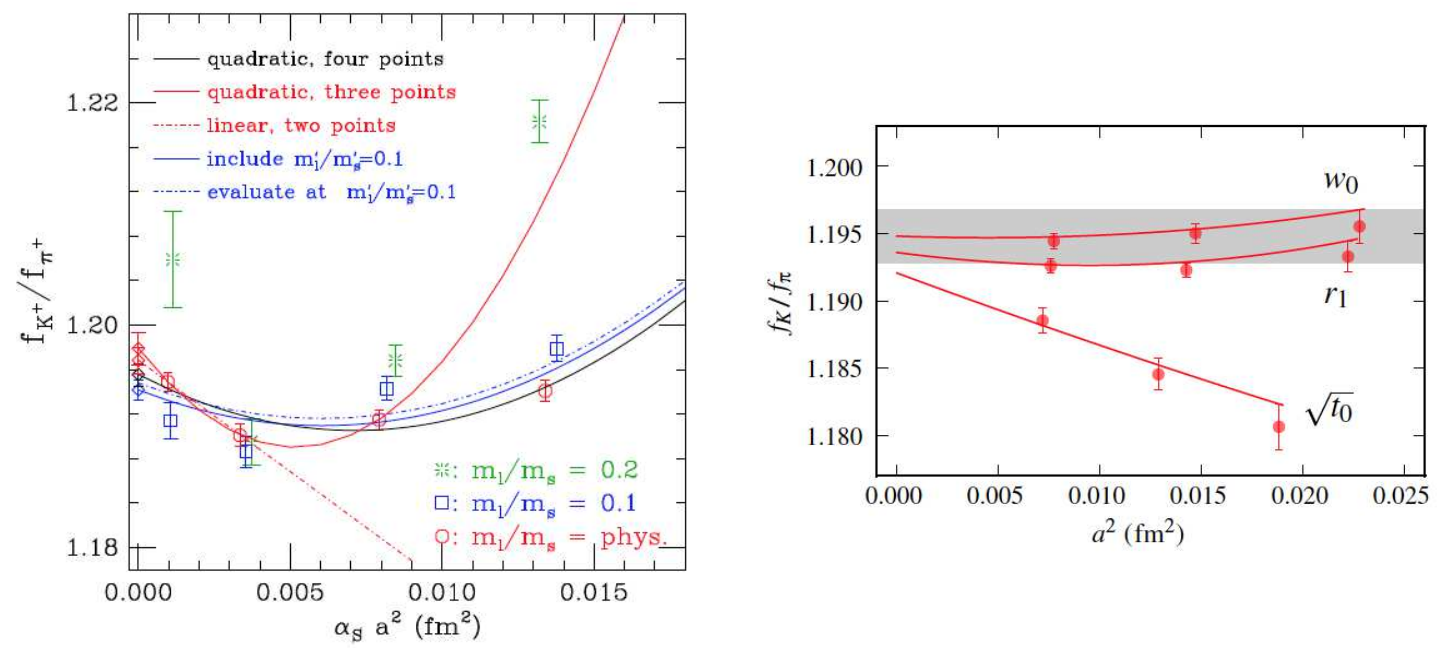

Figure 5: Continuum limit for the ratio of kaon and pion decay constants by FNAL/MILC (1.h.s.) and HPQCD (r.h.s.), respectively.

the continuum limits for the ratio of kaon and pion decay constants as obtained by FNAL/MILC and HPQCD. Both FNAL/MILC and HPQCD use overlapping sets of MILC's $N_{f}=2+1+1$ ensembles. Both collaborations include results for three lattice spacings $a=0.09,0.12,0.15 \mathrm{fm}$. FNAL/MILC however adds an even finer ensemble at $a=0.06 \mathrm{fm}$ allowing them to observe a significant upward turn towards the continuum limit relative to the data at $a=0.09 \mathrm{fm}$ and $0.12 \mathrm{fm}$ which was not anticipated by HPQCD's extrapolation from ensembles with $a \geq 0.09 \mathrm{fm}$. Likely as a result of this the final results by both collaboration are at variance:

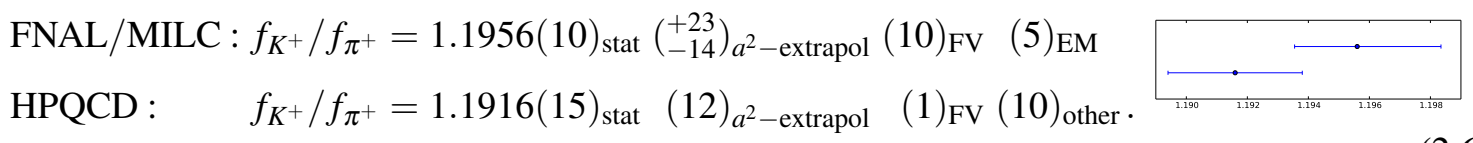

The tension amounts to about two standard deviations where it is crucial to keep in mind that both sets of results are statistically correlated due to the common set of underlying gauge ensembles. It should be interesting to see whether and how this apparent tension will be resolved in the future.

\subsection{Neutral kaon mixing}

The physical state $K_{L}$ consists predominantly of the $C P$-odd $K_{2}$ and a small admixture of the $C P$-even $K_{1}$. The decay of the $K_{2}$ into a $C P$-even two-pion state is called direct $C P$-violation and has been established experimentally in 1999 by NA48 and KTeV [21, 22]. Indirect $C P$-violation which occurs when the $K_{1}$ component in $K_{L}$ decays into the $C P$-even two-pion state has been established experimentally in 1964 by Cronin and Fitch [23]. Direct and indirect $C P$-violation have been studied on the lattice for many years and in the following I will discuss recent advances and results. See also Chris Kelly's talk at this conference [24]. The experimental measurement of $\left|\varepsilon_{K}\right|=\left|A\left(K_{L} \rightarrow(\pi \pi)_{I=0}\right) / A\left(K_{S} \rightarrow(\pi \pi)_{I=0}\right)\right|$ is confronted by the SM expression

$$
\varepsilon_{K}=e^{i \phi_{\varepsilon}} \cos \phi_{\varepsilon} \sin \phi_{\varepsilon}\left[\frac{\operatorname{Im} M_{12}}{2 \operatorname{Re} M_{12}}-\frac{\operatorname{Im} \Gamma_{12}}{2 \operatorname{Re} \Gamma_{12}}\right]
$$


where $\phi_{\varepsilon}$ is the weak phase, and $M$ and $\Gamma$ are the mass and decay matrix determining the timeevolution of neutral kaon mixing according to the Wigner-Weisskopf formula. In current phenomenological analyses the non-perturbative short-distance contribution to $\operatorname{Im} M_{12}$, by means of an operator product expansion, is expressed in terms of the kaon bag parameter

$$
\hat{B}_{K}=C(\mu) \frac{\left\langle\overline{K^{0}}\left|Q^{\Delta S=2}(\mu)\right| K^{0}\right\rangle}{\frac{8}{3} f_{K}^{2} m_{K}^{2}},
$$

which constitutes the leading term (see $[25,26]$ for a discussion of power counting in the OPE and also for the remaining ingredients in eqn. (2.7)). This equation then defines a hyperbola in the plane of the Wolfenstein parameters $\hat{\eta}$ and $\hat{\rho}$ which constrains the apex of the unitarity triangle. Here $Q^{\Delta S=2}(\mu)$ equals the difference of the parity even and parity odd four quark operators $O_{V V+A A}$ and $O_{V A+A V}$, respectively.

At this order of the OPE there are four more weak operators that mediate neutral kaon mixing beyond the $V-A$ structure found in the SM,

$$
\begin{array}{lll}
\mathscr{O}_{2}^{\Delta S=2}=\left(\bar{s}_{\alpha}\left(1-\gamma_{5}\right) d_{\alpha}\right)\left(\bar{s}_{\beta}\left(1-\gamma_{5}\right) d_{\beta}\right), & \mathscr{O}_{3}^{\Delta S=2}=\left(\bar{s}_{\alpha}\left(1-\gamma_{5}\right) d_{\beta}\right)\left(\bar{s}_{\beta}\left(1-\gamma_{5}\right) d_{\alpha}\right), \\
\mathscr{O}_{4}^{\Delta S=2}=\left(\bar{s}_{\alpha}\left(1-\gamma_{5}\right) d_{\alpha}\right)\left(\bar{s}_{\beta}\left(1+\gamma_{5}\right) d_{\beta}\right), & \mathscr{O}_{5}^{\Delta S=2}=\left(\bar{s}_{\alpha}\left(1-\gamma_{5}\right) d_{\beta}\right)\left(\bar{s}_{\beta}\left(1+\gamma_{5}\right) d_{\alpha}\right) .
\end{array}
$$

Their determination on the lattice is straight forward in principle and allows for model-independent constraints for $\Delta S=2$ new physics processes (e.g. discussion in [27]).

While all lattice results for the SM bag parameters are in nice agreement (see the preliminary 2016 FLAG scatter plot in figure 6), this is not the case for the BSM bag parameters, in particular

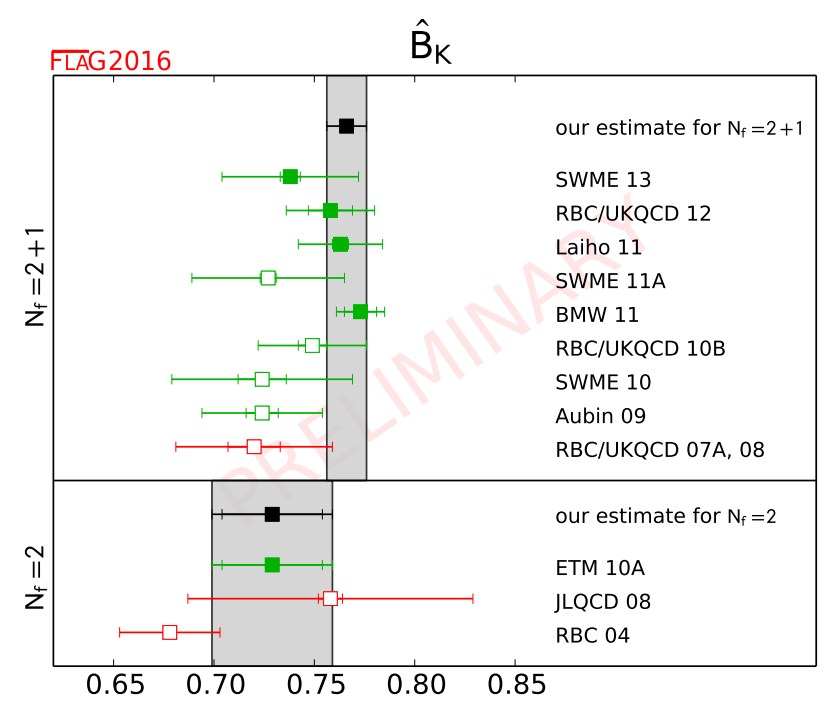

Figure 6: FLAG 2016 preliminary summary of lattice results for the (renormalisation group invariant) kaon bag parameter $\hat{B}_{K}$.

for $B_{2,4,5}$. An overview over current results is provided in figure 2.3. One can make the following observations: 
a) the results by ETM 12 [27] for $N_{f}=2$ and ETM 15 [28] and RBC/UKQCD 12 [29], respectively, are all consistent

b) the results by SWME 15 [30] and RBC/UKQCD 15 [31] are consistent

c) the results under a) and b) are under tension, if not incompatible.

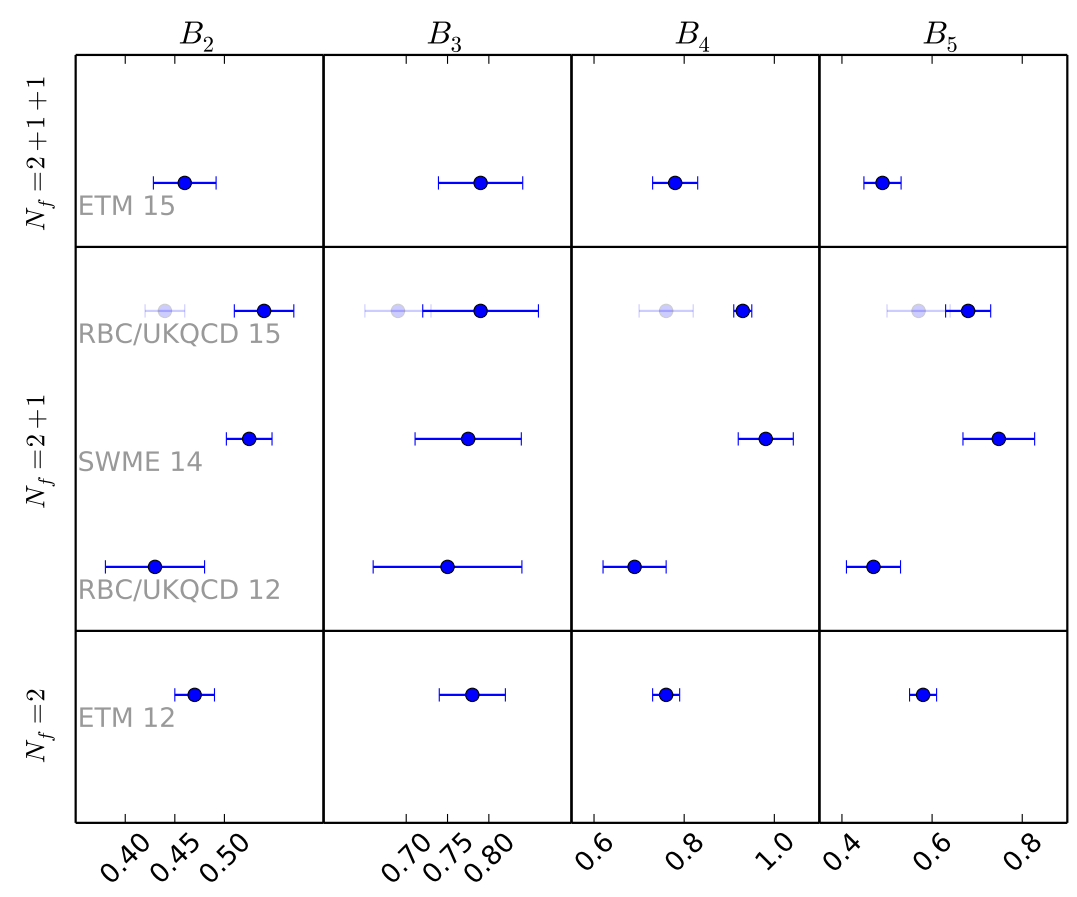

Figure 7: Summary of results for the BSM bag parameters $B_{2}-B_{5}$.

Clearly, ETM, RBC/UKQCD and SWME are, respectively, using different techniques in their computations, inducing different systematic uncertainties. Most striking, however, is the difference between RBC/UKQCD 12 and 15 since both results are from basically the same underlying gauge ensembles and therefore statistically correlated. The main difference between the two sets of results lies in the choice of intermediate renormalisation scheme (RI-MOM [32, 33] in RBC/UKQCD 12 and RI-SMOM [34, 35] in RBC/UKQCD 15). In RI-(S)MOM schemes the renormalisation condition is imposed on the operator's vertex function with external off-shell quark states projected on a particular choice of momentum. The induced momentum needs to be sufficiently large to be in the perturbative regime but at the same time small enough to keep momentum-dependent cutoff effects well under control. At low momenta in the RI-MOM scheme, however, non-perturbative effects e.g. a pion pole - substantially impact the shape of the vertex function. In $[34,36,37]$ however, it was found that for particular choices of the external momenta (non-exceptional momenta), the non-perturbative contributions at low momenta are suppressed or absent, thus removing a rather unpleasant source of systematic uncertainty. To illustrate this, figure 8 a) and b) show RBC/UKQCD's results for the quark mass dependence on the same ensemble of the vertex function of a 4-quark 


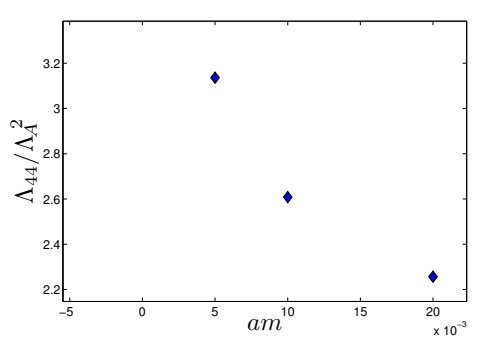

(a)

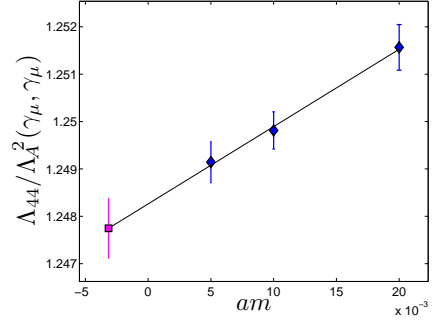

(b)

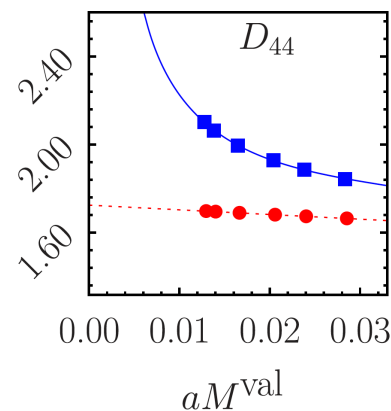

(c)

Figure 8: Mass dependence of vertex function. a) and c): RI-MOM by RBC/UKQCD and ETM, respectively; b): RI-SMOM scheme by RBC/UKQCD. Note the difference in resolution on the $y$-axis indicating the successful removal of the pion pole contribution in the SMOM scheme. Plots a) and b) courtesy of Nicolas Garron (RBC/UKQCD), c) from [28].

operator, normalised by the one of the axial current squared for both RI-MOM and RI-SMOM schemes, respectively. Note the vastly different scales on the $y$-axis. The data in plot a) seems to diverge for small quark masses which one explains with the contribution of a pion pole $\propto 1 / m_{\pi}^{2}$. This divergence is removed in plot b). Plot $c$ ) shows the corresponding results by the ETM collaboration in the RI-MOM scheme where again we see (blue data) the divergence appearing for small quark masses. ETM attempts to remove the divergence and extract a signal using the fit ansatz

$$
D_{i j}\left(a^{2} \tilde{p}^{2} ; m_{\mathrm{PS}}^{2}\right)=\mathscr{A}\left(a^{2} \tilde{p}^{2}\right)+\mathscr{B}\left(a^{2} \tilde{p}^{2}\right) m_{\mathrm{PS}}^{2}+\mathscr{C}\left(a^{2} \tilde{p}^{2}\right) / m_{\mathrm{PS}}^{2}+\mathscr{D}\left(a^{2} \tilde{p}^{2}\right) / m_{\mathrm{PS}}^{4}
$$

The data shown in red is the data after the pole-subtraction which turns out to be substantial.

In particular by comparing the two studies by the RBC/UKQCD collaboration one might suspect that systematic effects in the renormalisation could be a reason for the spread in results for the BSM bag parameters. Moreover, SWME, who renormalise the 4-quark operators perturbatively only, yield results that agree with RBC/UKQCD's RI-SMOM calculation. It will remain exciting to monitor how this puzzle (see also the discussion in [38]) will be resolved.

\subsection{Miscellaneous}

Heiri Leutwyler's appeal to the lattice community at Chiral Dynamics 2015, Pisa, stating "Please do not be content with reaching physical quark masses. Extract the dependence on them, determine the LECs!", has been responded to by at least two collaborations present at this conference: Firstly, RBC/UKQCD has carried out global NLO and NNLO analyses within $S U(2)$ and $S U(3)[39,40]$ chiral perturbation theory and extracted from it a comprehensive set of low energy constants.

Secondly, JLQCD [41] have carried out a global analysis of electromagnetic and semi-leptonic form factors on their $N_{f}=2+1$ overlap ensembles with fixed topological charge $Q$ at $a=0.11 \mathrm{fm}$. They are fitting $S U(3)$ NNLO chiral perturbation theory for which they use as input the low energy constants $L_{1-8}$ from [42]. They take advantage of the fact that the elm. and semileptonic form factors share certain LECs in order to reduce the overall number of degrees of freedom in the fit. Their preliminary result is $f_{+}^{K \rightarrow \pi}(0)=0.9636\left({ }_{-65}^{+62}\right)$. Note also ETM's new efforts in this direction [43, 44] on $N_{f}=2+1+1$ ensembles of twisted mass fermions at three different values 
of the lattice spacing and with a comprehensive study of the dependence of the form factor on the momentum transfer $q$.

One result presented at this conference and worth highlighting is a renewed effort by ETM to complete their set of $N_{f}=2$ flavour ensembles. Most collaborations have moved on to simulations of $N_{f}=2+1(+1)$ since they are more useful for SM phenomenology. This has lead to a situation where no state-of-the-art results for $N_{f}=2$ with comparable control of systematic uncertainties (in particular, not with physical light quark masses) exists. Having $N_{f}=2$ results would however still be highly interesting. For instance, the $N_{f}$ dependence of low-energy quantities is not yet fully understood, low energy constants for the $N_{f}=2$ theory could be determined more reliably, ... . I hope that ETM will push this study further and provide the set of reference results for $N_{f}=2$.

\section{Beyond standard quantities}

\subsection{Including QED effects in hadronic matrix elements}

Current phenomenological analyses of kaon and pion decays are based on equations (2.1) and (2.2). In practice the decay constant or the form factor are computed in isospin symmetric QCD. Strong isospin is corrected for either through chiral perturbation theory (FLAG for instance is doing this [2]) or by applying a correction based on the valence quark mass dependence of the decay constant or form factor known in terms of the fit to lattice data (MILC for instance is doing this [19]). In all cases QED effects are corrected for using the formulae provided by effective theory.

The precision that can now be achieved in lattice QCD simulations of pion and kaon decay constants and form factors is comparable to the size of electromagnetic and strong isospin corrections. It therefore appears timely and natural to try and replace the model and effective theory based estimates by direct predictions on the lattice. How this can be done for spectral quantities has been summarised by Antonin Portelli at the 2014 Lattice conference [45]. At the same conference, also first ideas on how to extend such computations towards hadronic matrix elements were presented by Chris Sachrajda $[46,47]$.

While leptonic decays are well parametrised at $O\left(\alpha^{0}\right)$ as

$$
\Gamma\left(\pi^{+} \rightarrow l^{+} v_{l}\right)=\frac{G_{F}^{2}\left|V_{u d}\right|^{2} f_{\pi}^{2}}{8 \pi} m_{\pi} m_{l}^{2}\left(1-\frac{m_{l}^{2}}{m_{\pi}^{2}}\right)^{2},
$$

at $O(\alpha)$, IR divergences arise in individual diagrams that contribute but eventually cancel in the sum of the diagrams (Bloch-Nordsieck [48]). In particular, the individual terms on the r.h.s. of

$$
\Gamma\left(\pi^{+} \rightarrow l^{+} v_{l}(\gamma)\right)=\Gamma\left(\pi^{+} \rightarrow l^{+} v_{l}\right)+\Gamma\left(\pi^{+} \rightarrow l^{+} v_{l} \gamma\right)
$$

diverge, where $\Gamma_{0}$ contains all diagrams with only internal photon lines and $\Gamma_{1}$ contains a photon in the final state. The idea for how to handle the IR divergences in a lattice simulation was presented in [47] and the authors suggest an analysis where only experimental data with a cut on the photon momenta $\Delta E<20 \mathrm{MeV}$ has been applied. The rationale is that in this situation the outgoing photon merely sees a particle that can be well approximated by a point-particle. In this situation,

$$
\Gamma(\Delta E)=\lim _{V \rightarrow \infty}\left(\Gamma_{0}-\Gamma_{0}^{\mathrm{pt}}\right)+\lim _{V \rightarrow \infty}\left(\Gamma_{0}^{\mathrm{pt}}+\Gamma_{1}^{\mathrm{pt}}(\Delta E)\right) .
$$


The first term on the r.h.s. contains $\Gamma_{0}$ obtained from a lattice computation with only internal photon lines from which the same process in finite volume $V$ computed in perturbation theory in the point-like approximation is subtracted. The crucial observation is that this difference is IR finite since at long distance both terms describe the same physics. Also finite volume effects are expected to be the same for both terms. They can be computed analytically or be extrapolated away in a series of simulations with different volumes. The contributions to the second term on the r.h.s. can be computed in the point-like approximation in perturbation theory in infinite volume. This second term is again IR finite. Part of the work has been reported on in [47] and at this conference an update on the numerical implementation was presented. In particular, the computation of the diagrams in figure 9, which involves evaluating correlation functions of the form

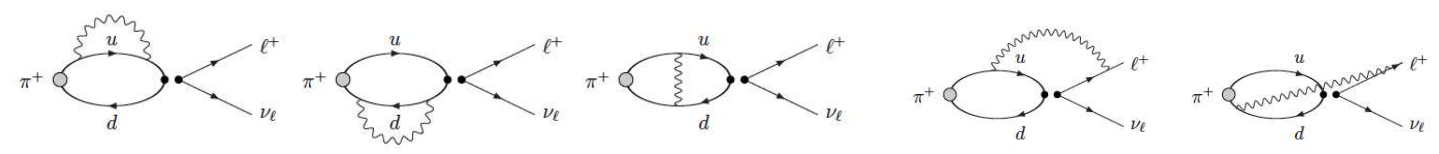

Figure 9: Class of diagrams to be evaluated for the computation of $\Gamma_{0}$.

$$
C_{1}(t)=-\frac{1}{2} \int d^{3} \vec{x} d^{4} x_{1} d^{4} x_{2}\left\langle 0\left|T\left\{J_{W}^{v}(0) j_{\mu}\left(x_{1}\right) j_{\mu}\left(x_{2}\right) \phi^{\dagger}(\vec{x},-t)\right\}\right| 0\right\rangle \Delta\left(x_{1}, x_{2}\right),
$$

and

$$
\begin{aligned}
C_{1}(t)_{\alpha \beta}=- & \int d^{3} \vec{x} d^{4} x_{1} d^{4} x_{2}\left\langle 0\left|T\left\{J_{W}^{v}(0) j_{\mu}\left(x_{1}\right) \phi^{\dagger}(\vec{x},-t)\right\}\right| 0\right\rangle \\
& \times \Delta\left(x_{1}, x_{2}\right)\left(\gamma_{v}\left(1-\gamma^{5}\right) S\left(0, x_{2}\right) \gamma_{\mu}\right)_{\alpha \beta} e^{E_{l} t_{2}-i \vec{p}_{l} \cdot \vec{x}_{2}},
\end{aligned}
$$

with explicit insertions of the photon and lepton propagators, respectively. First results are illustrated in figure 10 for various combinations of currents between the pion and the vacuum. The plots show that a signal can be obtained for the type of correlation functions in eqns. (3.4) and (3.5). This work constitutes the first attempt at a conceptually clean calculation in lattice QCD of leptonic decays including $O(\alpha)$ QED effects. The next step in this project will be the evaluation of quark-disconnected contributions and combining the numerical data with the analytical predictions for $\Gamma_{0}^{\mathrm{pt}}$ and $\Gamma_{1}^{\mathrm{pt}}(\Delta E)$ as indicated in eqn. (3.3). A statistical error of the order of $20 \%$ on the QED contributions would be sufficient for use for phenomenology and might be feasible in the not so distant future.

\subsection{Long distance effects}

The error budget of current uses of $\varepsilon_{K}$ in flavour phenomenology is dominated by the uncertainty in $\left|V_{c b}\right|$ entering eqn. (2.7) via the box-diagram mediating the mixing. Still, depending on the choice of input to the analysis, in particular whether $\left|V_{c b}\right|$ is taken from exclusive or inclusive determinations, one sees either a $3.4 \sigma$ tension between the prediction for $\varepsilon_{K}$ and the experimental value [20], or none, as highlighted in Lee's poster [49, 50].

The lattice prediction for the kaon bag parameter is now well controlled and possible with a precision of around $1 \%[2,3]$. The bag parameter at this point no longer constitutes the dominant non-perturbative uncertainty. This role has now been taken over by long-distance effects, as 

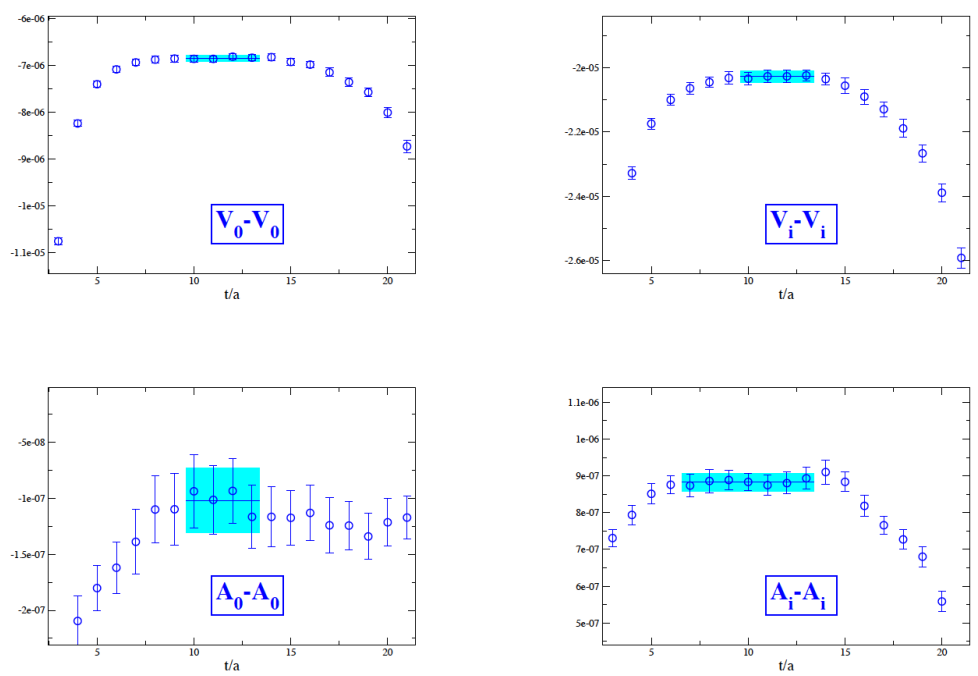

Figure 10: First numerical results for the correlation functions entering the computation of $\Gamma_{0}$.

anticipated in $[25,26]$. When lifting the prediction for $\varepsilon_{K}$ to the next order in the OPE, long distance effects in both $\operatorname{Im} M_{12}$ and $\operatorname{Im} \Gamma_{12}$ need to be taken into account. Long distance effects can contribute to CP violation in kaon mixing at the level of 5\% [26] and therefore deserve attention.

The long distance contribution originates from two insertions of dimension-six $\Delta S=1$ effective weak operators,

$$
\mathscr{T}_{12}=-i \int d^{4} x\left\langle K^{0}\left|T\left[\mathscr{H}_{|\Delta S|=1}^{(u, c)}(x) \mathscr{H}_{|\Delta S|=1}^{(u, c)}(0)\right]\right| \bar{K}^{0}\right\rangle
$$

Beyond $\varepsilon_{K}$, there is wider interest in reliable predictions of matrix elements with two insertions of effective weak or electromagnetic operators between initial and final hadronic states: For instance, compared to typical QCD scales the closely related $K_{L}-K_{S}=2 \mathrm{Re} M_{12}$ mass difference, known experimentally to be $\Delta M_{K}=3.484(6) \times 10^{-12} \mathrm{MeV}$ [20], is a tiny quantity and therefore a sensitive probe for new physics. Long distance effects to it should be around 20\% [51]. If predicted reliably at the level of $10 \%$ precision it could be a probe for new physics at scales up to $O(\mathrm{TeV})$ [52].

In a sense the above-mentioned correlation functions relevant for the computation of QED effects in hadronic matrix elements, eqns. (3.4) and (3.5) are of a similar type. Later in this article we will also briefly consider rare kaon decays where they play a role. Following $[53,52]$ the

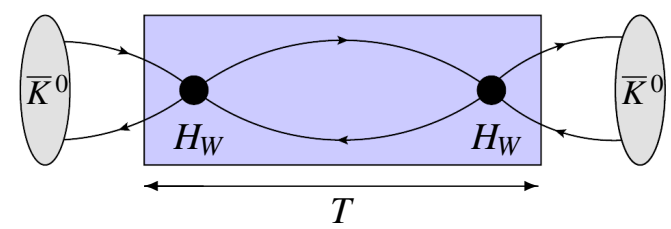

Figure 11: Kaon mixing with two insertions of dimension-six $\Delta_{S}=1$ weak operators. 
amplitude in eqn. (3.6) can be computed in Euclidean space from

$$
\mathscr{A}=\left\langle 0\left|T\left\{K^{0}\left(t_{f}\right) \frac{1}{2} \int_{t_{A}}^{t_{B}} d t_{2} \int_{t_{A}}^{t_{B}} d t_{1} H_{W}\left(t_{2}\right) H_{W}\left(t_{1}\right) K^{0^{\dagger}}\left(t_{i}\right)\right\}\right| 0\right\rangle,
$$

where the integration over Euclidean time is taken as $t_{B}-t_{A}=T-1$ with $t_{A}$ and $t_{B}$ such that only the kaon ground state contributes. The amplitude can be evaluated to be

$$
\mathscr{A}=N_{K}^{2} e^{-M_{K}\left(t_{f}-t_{i}\right)} \sum_{n} \frac{\left\langle\bar{K}^{0}\left|H_{W}\right| n\right\rangle\left\langle n\left|H_{W}\right| K^{0}\right\rangle}{M_{K}-E_{n}}\left(-T-\frac{1}{M_{K}-E_{n}}+\frac{e^{\left(M_{K}-E_{n}\right) T}}{M_{K}-E_{n}}\right) .
$$

The term linear in $T$ corresponds to the actual physical amplitude, the second term is a constant that needs to be subtracted. The remaining contribution is either exponentially decreasing(increasing) if $E_{n}>M_{K}\left(E_{n}<M_{K}\right)$ for the hadronic state $n$ mediating between the two $H_{W}$. For instance, an intermediate vacuum-, $\pi^{0}$ - or $\eta$-state need to be subtracted in order to extract the actual signal. To this end several methods are being studied [53, 52, 54]. For instance, the contributions can be determined from a fit to the $T$-dependence of the correlation function. Or, the matrix elements $\left\langle n\left|H_{W}\right| K^{0}\right\rangle$ can be computed separately from suitably constructed meson $3 \mathrm{pt} \mathrm{functions.} \mathrm{Yet} \mathrm{another}$ option is to add terms $\bar{s} d$ or $\bar{s} \gamma_{5} d$ to the weak Hamiltonian with prefactors that can be computed to explicitly subtract unwanted states. These additional terms, by means of the Vector and Axial Ward Identity, are total derivatives and do not modify the physics. For instance, for the subtraction of the vacuum and $\eta$ intermediate states one determines the prefactors $c_{S}$ and $c_{P}$ for the additional terms to the effective Hamiltonian from

$$
\left\langle 0\left|H_{W}+c_{P} \bar{s} \gamma_{5} d\right| K\right\rangle=0 \quad \text { and } \quad\left\langle\eta\left|H_{W}+c_{S} \bar{s} d\right| K\right\rangle=0 .
$$

Given that multi-hadron intermediate states can contribute to $\mathscr{A}$ special care needs to be taken to control finite volume effects [55, 56]. Care also has to be taken for $x \rightarrow 0$. In the case of $\Delta M_{K}$, GIM-cancellation together with the $V-A$ structure of the weak operator ensure that no residual divergence has to be dealt with. In the case of $\varepsilon_{K}$ a residual logarithmic divergence has to be removed. Exploratory studies for both quantities are under way [52, 57].

Another class of processes with important long distance contributions are rare kaon decays. Two new experiments are dedicated to the study of these decays: NA62 at CERN and KOTO at J-PARC. As flavour changing neutral current processes they constitute a probe into flavour mixing and BSM physics due to their suppression relative to the SM.

Only a subset of the various rare kaon decay channels have sufficiently large long distance contributions to warrant a lattice QCD study. Amongst those are the CP-conserving decays $K_{S} \rightarrow$ $\pi^{0} v \bar{v}, K^{+} \rightarrow \pi^{+} v \bar{v}, K_{S} \rightarrow \pi^{0} l^{+} l^{-}$and $K^{+} \rightarrow \pi^{+} l^{+} l^{-}$. This last decay is illustrated diagrammatically in figure 12. Current experimental facilities are able in principle to study this channel as well. The decay into charged leptons is long-distance dominated through one-photon exchange and has previously been investigated in chiral perturbation theory [58, 59]. A first principles calculation of the form factor in lattice QCD, besides its use in flavour phenomenology $\left(V_{t s}\right.$ and $\left.V_{t d}\right)$ will therefore also constitute a test of chiral effective theory.

Considering as an example the decay $K^{+} \rightarrow \pi^{+} l^{+} l^{-}$, the amplitude to be computed is similar in structure to the one for $\varepsilon_{K}$ and $\Delta M_{K}$ in eq. (3.7) but with one of the effective weak Hamiltonians 


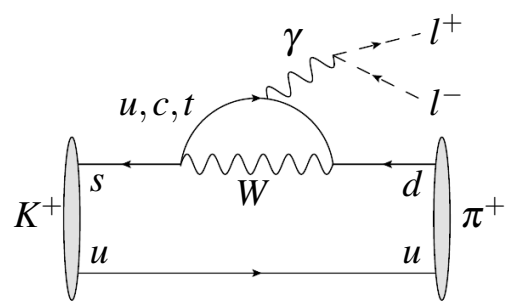

Figure 12: Diagrams for $K^{+} \rightarrow \pi^{+} l^{+} l^{-}$kaon semileptonic rare decays.

replaced by a conserved vector current representing the coupling to the photon [60, 61, 54],

$$
\mathscr{A}_{\mu}=\left(q^{2}\right) \int d^{4} x\left\langle\pi(p)\left|T\left[J_{\mu}(0) H_{W}(x)\right]\right| K(k)\right\rangle \text {. }
$$

The two effective weak operators $Q_{1}^{q}=\left(\bar{s}_{i} \gamma_{\mu}^{L} d_{i}\right)\left(\bar{q}_{j} \gamma_{\mu}^{L} q_{j}\right)$ and $Q_{2}^{q}=\left(\bar{s}_{i} \gamma_{\mu}^{L} q_{i}\right)\left(\bar{q}_{j} \gamma_{\mu}^{L} d_{j}\right)$, respectively, provide for the dominant contributions. When integrating the weak operator over the fiducial volume (cf. figure 11 and eq. 3.7) gauge invariance and the GIM mechanism remove residual divergences. One yet again identifies exponentially growing and decaying terms which are unphysical and hence have to be subtracted, in particular, $\pi, \pi \pi$ (can be shown not to contribute by symmetry) and $\pi \pi \pi$ (expected to be small). In a pilot study RBC/UKQCD have set out to compute the corresponding form factor $V_{i}$ defined as

$$
A_{i}=-\frac{G_{F} \alpha}{4 \pi} V_{i}(z)(k+p)^{\mu} \bar{u}_{l}\left(p_{-}\right) \gamma_{\mu} v_{l}\left(p_{+}\right)
$$

and presented first results at this conference [54]. For their unphysical kinematics with $m_{\pi} \approx$ $421 \mathrm{MeV}$ and $m_{K}=600 \mathrm{MeV}$, they were able to obtain a signal after successfully subtracting the one-pion intermediate state contribution (the only allowed intermediate state for this choice of kinematics). The subtraction and integration over the fiducial volume are illustrated in the series of plots in figure 13 .

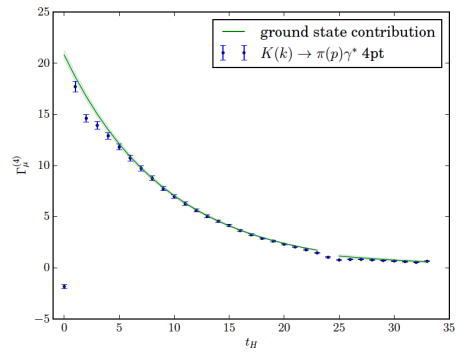

(a)

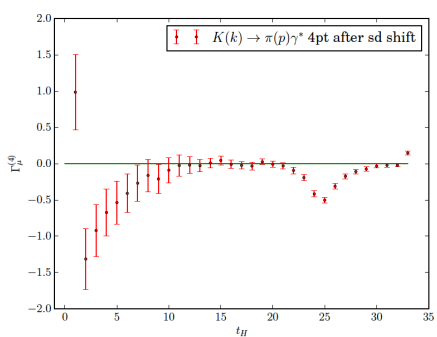

(b)

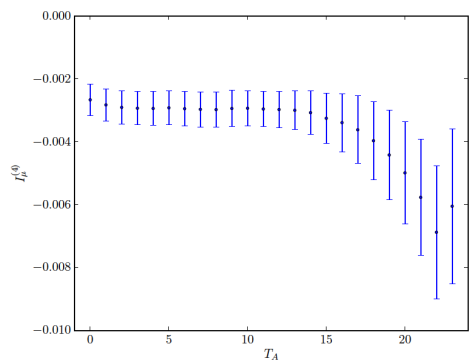

(c)

Figure 13: Example for $K \rightarrow \pi \gamma^{*}$ correlation function in (a), after subtraction of $\pi$-intermediate state (b), after integration over fiducial volume (c).

The decay $K^{+} \rightarrow \pi^{+} v \bar{v}$ is harder by the fact that both photon and Z-exchange diagrams as well as $W W$-diagrams contribute (cf. figure 14). Power divergences are only partially removed leaving a logarithmic divergence that needs to be taken care of [62]. 


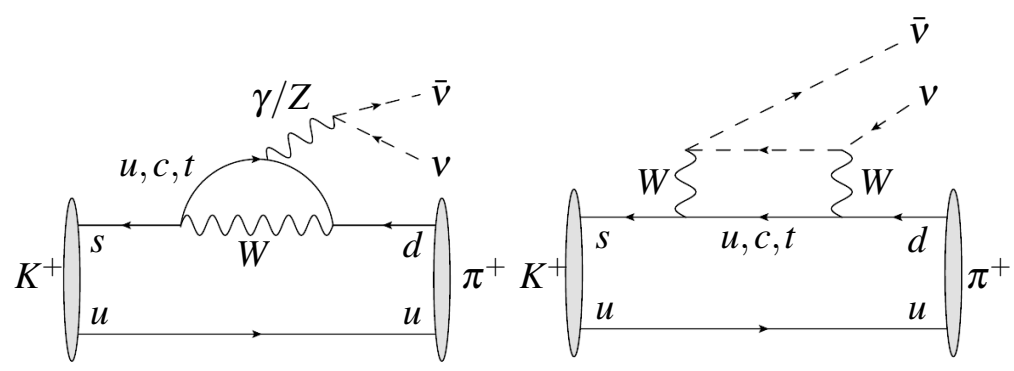

Figure 14: Diagrams for $K^{+} \rightarrow \pi^{+} v \bar{v}$ kaon semileptonic rare decays.

\section{Timelike form factors}

Spacelike momenta are the natural domain for simulations of e.g. the pion form factor on a Euclidean lattice. The timelike region is however still of considerable phenomenological interest for e.g. low-energy experiments and chiral perturbation theory. To this end Meyer [63] derived a relation between the absolute value of the $\pi \pi$ to vacuum matrix element of the electromagnetic current, to the pion form factor in the timelike region. The relation was extended to the moving frame in [64] allowing to map out the form factor in the whole elastic $\pi \pi$ scattering region. That same work also presents a first numerical study with $N_{f}=2+1$ overlap quarks (fixed topology, $\left.m_{\pi}>290 \mathrm{MeV}\right)$. They obtained a good signal for the form factor and in addition extracted the coupling $g_{\rho \pi \pi}$ and the $\rho$-mass, cf. figure 15 . In this context also have a look at the contributions
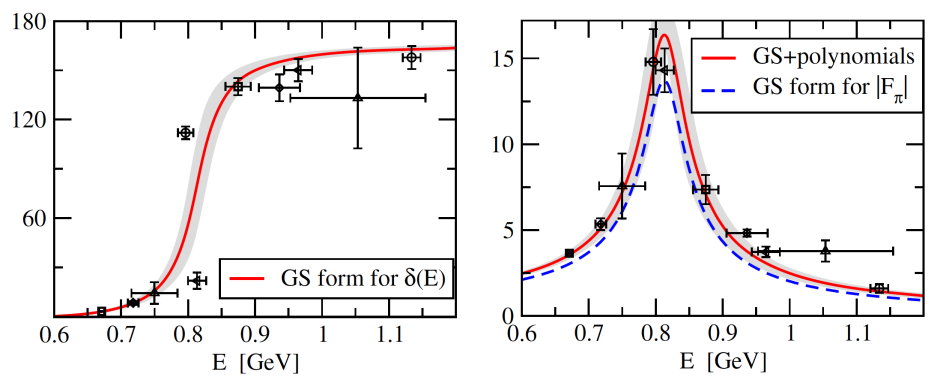

Figure 15: Results for the scattering phases (left) and modulus of the pion form factor (right) as computed in [64].

by Werner [65] on the $\rho$ resonance parameters.

\section{Conclusions}

Simulations with the light quark masses tuned such that pion masses assume the value found in nature allow for a new quality of simulation results and at the same time open the door for new data analysis strategies. In particular those analysis strategies that minimise model dependencies (here the model for the quark mass dependence) can be devised. In this way long chiral extrapolations are no longer necessary and no longer play a role in systematic error budgets. Instead, statistical errors, finite volume corrections and the continuum extrapolation error tend to dominate error budgets. 
Kaon and pion decay constants and semi-leptonic form factors which are crucial input for first-row CKM phenomenology can now predicted reliably with sub-percent level precision. Since this is the expected size of isospin breaking effects, new methods are now being devised to include these in lattice simulations.

I highlighted an inconsistency between computations of BSM kaon mixing matrix elements which could potentially be explained with an underestimated systematic effect in the non-perturbative renormalisation. Having several independent computations of the same quantity was very helpful in identifying this apparent discrepancy.

The remainder of the talk was dedicated to conceptually new efforts: the computation of hadronic matrix elements with two insertions of electroweak and electromagnetic operators. Examples are $\Delta M_{K}, \varepsilon_{K}$ and rare kaon decays, each of which opens the door to a variety of phenomenological applications. These quantities are particularly sensitive to BSM phsyics.

Last but not least I presented the application of a new theoretical idea to compute form factors in the timelike regime from Euclidean correlation functions.

Acknowledgements: The author would like to thank the organisers of this perfectly prepared and managed conference and acknowledges funding from the European Research Council under the European Union's Seventh Framework Programme (FP7/2007-2013) / ERC Grant agreement 279757.

\section{References}

[1] G. Colangelo et. al., Review of lattice results concerning low energy particle physics, Eur. Phys. J. C71 (2011) 1695, [1011.4408].

[2] S. Aoki et. al., Review of lattice results concerning low-energy particle physics, Eur. Phys. J. C74 (2014) 2890, [1310.8555].

[3] In preparation, Review of lattice results concerning low-energy particle physics, .

[4] W. J. Marciano, Precise determination of $\left|V_{u s}\right|$ from lattice calculations of pseudoscalar decay constants, Phys. Rev. Lett. 93 (2004) 231803, [hep-ph/ 0402299 ].

[5] FlaviaNet Working Group on Kaon Decays Collaboration, M. Antonelli et. al., An Evaluation of $\left|V_{u s}\right|$ and precise tests of the Standard Model from world data on leptonic and semileptonic kaon decays, Eur. Phys. J. C69 (2010) 399-424, [1 005 .2323].

[6] J. Gasser and H. Leutwyler, Chiral Perturbation Theory: Expansions in the Mass of the Strange Quark, Nucl. Phys. B250 (1985) 465-516.

[7] V. Cirigliano and H. Neufeld, A note on isospin violation in $P_{l 2}(\gamma)$ decays, Phys. Lett. $\mathbf{B 7 0 0}$ (2011) $7-10,[1102.0563]$.

[8] M. Moulson, Experimental determination of $V_{u s}$ from kaon decays, in 8th International Workshop on the CKM Unitarity Triangle (CKM2014) Vienna, Austria, September 8-12, 2014, 2014. 1411.5252.

[9] V. Cirigliano, M. Giannotti, and H. Neufeld, Electromagnetic effects in $K_{l 3}$ decays, JHEP 11 (2008) 006, [0807.4507].

[10] J. Gasser and H. Leutwyler, Low-Energy Expansion of Meson Form-Factors, Nucl. Phys. B250 (1985) $517-538$. 
[11] V. Cirigliano, G. Ecker, H. Neufeld, A. Pich, and J. Portoles, Kaon Decays in the Standard Model, Rev. Mod. Phys. 84 (2012) 399, [1107.6001].

[12] RBC/UKQCD Collaboration, P. A. Boyle et. al., The kaon semileptonic form factor in $N_{f}=2+1$ domain wall lattice QCD with physical light quark masses, JHEP 06 (2015) 164, [1504. 01692 ].

[13] H. Na, C. T. H. Davies, E. Follana, G. P. Lepage, and J. Shigemitsu, The $D \rightarrow K$, lv Semileptonic Decay Scalar Form Factor and $\left|V_{c s}\right|$ from Lattice QCD, Phys. Rev. D82 (2010) 114506, [1008.4562].

[14] M. Ademollo and R. Gatto, Nonrenormalization Theorem for the Strangeness Violating Vector Currents, Phys. Rev. Lett. 13 (1964) 264-265.

[15] RBC, UKQCD Collaboration, T. Blum et. al., Domain wall QCD with physical quark masses, 1411.7017.

[16] A. Roessl, Pion kaon scattering near the threshold in chiral SU(2) perturbation theory, Nucl. Phys. B555 (1999) 507-539, [hep-ph/ 9904230$].$

[17] RBC-UKQCD Collaboration, C. Allton et. al., Physical Results from 2+1 Flavor Domain Wall QCD and SU(2) Chiral Perturbation Theory, Phys. Rev. D78 (2008) 114509, [0 804.0473 ].

[18] R. J. Dowdall, C. T. H. Davies, G. P. Lepage, and C. McNeile, $V_{u s}$ from $\pi$ and $K$ decay constants in full lattice QCD with physical $u, d$, s and c quarks, Phys. Rev. D88 (2013) 074504, [1303. 1670 ].

[19] Fermilab Lattice, MILC Collaboration, A. Bazavov et. al., Charmed and light pseudoscalar meson decay constants from four-flavor lattice QCD with physical light quarks, Phys. Rev. D90 (2014), no. 7 074509, [1407.3772].

[20] Particle Data Group Collaboration, K. A. Olive et. al., Review of Particle Physics, Chin. Phys. C38 (2014) 090001.

[21] NA48 Collaboration, V. Fanti et. al., A new measurement of direct CP violation in two pion decays of the neutral kaon, Phys. Lett. B465 (1999) 335-348, [hep-ex/9909022].

[22] KTeV Collaboration, A. Alavi-Harati et. al., Observation of direct CP violation in $K_{S, L} \rightarrow \pi \pi$ decays, Phys. Rev. Lett. 83 (1999) 22, [hep-ex/9905060].

[23] J. H. Christenson, J. W. Cronin, V. L. Fitch, and R. Turlay, Evidence for the $2 \pi$ decay of the $K_{2}^{0}$ meson, Phys. Rev. Lett. 13 (1964) 138-140.

[24] In these proceedings, C. Kelly, Standard-model prediction for direct CP violation in $K \rightarrow \pi \pi$ decays, .

[25] A. J. Buras and D. Guadagnoli, Correlations among new CP violating effects in $\Delta F=2$ observables, Phys. Rev. D78 (2008) 033005, [0 805 .3887].

[26] A. J. Buras, D. Guadagnoli, and G. Isidori, On $\varepsilon_{K}$ Beyond Lowest Order in the Operator Product Expansion, Phys. Lett. B688 (2010) 309-313, [1 002 . 3612].

[27] ETM Collaboration, V. Bertone et. al., Kaon Mixing Beyond the SM from $N_{f}=2$ tmQCD and model independent constraints from the UTA, JHEP 03 (2013) 089, [1207 . 1287]. [Erratum: JHEP07,143(2013)].

[28] ETM Collaboration, N. Carrasco, P. Dimopoulos, R. Frezzotti, V. Lubicz, G. C. Rossi, S. Simula, and C. Tarantino, $\Delta S=2$ and $\Delta C=2$ bag parameters in the standard model and beyond from $N_{f}=2+1+1$ twisted-mass lattice QCD, Phys. Rev. D92 (2015), no. 3 034516, [1505. 0663 9].

[29] RBC, UKQCD Collaboration, R. Arthur et. al., Domain Wall QCD with Near-Physical Pions, Phys. Rev. D87 (2013) 094514, [1208.4412]. 
[30] SWME Collaboration, B. J. Choi et. al., Kaon BSM B-parameters using improved staggered fermions from $N_{f}=2+1$ unquenched QCD, Phys. Rev. D93 (2016), no. 1 014511, [1509.00592].

[31] R. J. Hudspith, N. Garron, and A. T. Lytle, Neutral Kaon mixing beyond the Standard Model, 2015. 1512.05398.

[32] G. Martinelli, S. Petrarca, C. T. Sachrajda, and A. Vladikas, Nonperturbative renormalization of two quark operators with an improved lattice fermion action, Phys. Lett. B311 (1993) 241-248. [Erratum: Phys. Lett.B317,660(1993)].

[33] G. Martinelli, C. Pittori, C. T. Sachrajda, M. Testa, and A. Vladikas, A General method for nonperturbative renormalization of lattice operators, Nucl. Phys. B445 (1995) 81-108, [hep-lat/9411010].

[34] C. Sturm, Y. Aoki, N. H. Christ, T. Izubuchi, C. T. C. Sachrajda, and A. Soni, Renormalization of quark bilinear operators in a momentum-subtraction scheme with a nonexceptional subtraction point, Phys. Rev. D80 (2009) 014501, [0 901.2599 ].

[35] Y. Aoki et. al., Non-perturbative renormalization of quark bilinear operators and $B_{K}$ using domain wall fermions, Phys. Rev. D78 (2008) 054510, [0712.1061].

[36] RBC, UKQCD Collaboration, Y. Aoki et. al., Continuum Limit Physics from 2+1 Flavor Domain Wall QCD, Phys. Rev. D83 (2011) 074508, [1 011.0892$].$

[37] Y. Aoki et. al., Continuum Limit of $B_{K}$ from 2+1 Flavor Domain Wall QCD, Phys. Rev. D84 (2011) 014503, [1 012 . 4178].

[38] N. Garron, CP violation and Kaon weak matrix elements from Lattice $Q C D$, in 8th International Workshop on Chiral Dynamics (CD 2015) Pisa, Italy, June 29-July 3, 2015, 2015. 1512.02440.

[39] P. A. Boyle et. al., Low energy constants of $S U(2)$ partially quenched chiral perturbation theory from $N_{f}=2+1$ domain wall QCD, Phys. Rev. D93 (2016), no. 5 054502, [1511.01950].

[40] R. D. Mawhinney and D. J. Murphy, NLO and NNLO Low Energy Constants for SU (2) Chiral Perturbation Theory, in Proceedings, 33rd International Symposium on Lattice Field Theory (Lattice 2015), 2015. 1511.04419.

[41] JLQCD Collaboration, S. Aoki, G. Cossu, X. Feng, S. Hashimoto, T. Kaneko, J. Noaki, and T. Onogi, Light meson electromagnetic form factors from three-flavor lattice QCD with exact chiral symmetry, 1510.06470 .

[42] J. Bijnens and G. Ecker, Mesonic low-energy constants, Ann. Rev. Nucl. Part. Sci. 64 (2014) 149-174, [1405.6488].

[43] N. Carrasco, P. Lami, V. Lubicz, L. Riggio, and S. Simula, Momentum dependence of kaon semileptonic form factors with $N_{f}=2+1+1$ Twisted Mass fermions, in Proceedings, 33rd International Symposium on Lattice Field Theory (Lattice 2015), 2015. 1511.04880.

[44] N. Carrasco, P. Lami, V. Lubicz, L. Riggio, S. Simula, and C. Tarantino, $K \rightarrow \pi$ semileptonic form factors with $N_{f}=2+1+1$ Twisted Mass fermions, 1602.04113 .

[45] A. Portelli, Inclusion of isospin breaking effects in lattice simulations, PoS LATTICE2014 (2015) 013, [1505.07057].

[46] C. T. Sachrajda, Long-distance contributions to flavour-changing processes, PoS LATTICE2014 (2015) 023, [1503.01691]. 
[47] N. Carrasco, V. Lubicz, G. Martinelli, C. T. Sachrajda, N. Tantalo, C. Tarantino, and M. Testa, QED Corrections to Hadronic Processes in Lattice QCD, Phys. Rev. D91 (2015), no. 7074506 , [1502.00257].

[48] F. Bloch and A. Nordsieck, Note on the Radiation Field of the electron, Phys. Rev. 52 (1937) 54-59.

[49] SWME Collaboration, J. A. Bailey, Y.-C. Jang, W. Lee, and S. Park, Standard Model evaluation of $\varepsilon_{K}$ using lattice $Q C D$ inputs for $\hat{B}_{K}$ and $V_{c b}$, Phys. Rev. D92 (2015), no. 3 034510, [1503. 05388 ].

[50] J. A. Bailey, Y.-C. Jang, W. Lee, and S. Park, Determination of $\varepsilon_{K}$ using lattice QCD inputs, PoS LATTICE2015 (2015) 348, [1511.00969].

[51] S. Herrlich and U. Nierste, Enhancement of the $K_{L}-K_{S}$ mass difference by short distance $Q C D$ corrections beyond leading logarithms, Nucl. Phys. B419 (1994) 292-322, [hep-ph/9310311].

[52] Z. Bai, N. H. Christ, T. Izubuchi, C. T. Sachrajda, A. Soni, and J. Yu, $K_{L}-K_{S}$ Mass Difference from Lattice QCD, Phys. Rev. Lett. 113 (2014) 112003, [1406.0916].

[53] RBC, UKQCD Collaboration, N. H. Christ, Computing the long-distance contribution to second order weak amplitudes, PoS LATTICE2010 (2010) 300.

[54] N. Christ, X. Feng, A. Juttner, A. Lawson, A. Portelli, and C. Sachrajda, Long distance contributions to the rare kaon decay $K \rightarrow \pi \ell^{+} \ell^{-}$, in Proceedings, 33rd International Symposium on Lattice Field Theory (Lattice 2015), 2016. 1602.01374.

[55] N. H. Christ, X. Feng, G. Martinelli, and C. T. Sachrajda, Effects of finite volume on the $K_{L}-K_{S}$ mass difference, Phys. Rev. D91 (2015), no. 11 114510, [1504.01170].

[56] R. A. Briceño and M. T. Hansen, Multichannel $0 \rightarrow 2$ and $1 \rightarrow 2$ transition amplitudes for arbitrary spin particles in a finite volume, Phys. Rev. D92 (2015), no. 7 074509, [1502.04314].

[57] In these proceedings, Z. Bai and N. Christ, Computing the long distance ontributions to $\varepsilon_{K}$, .

[58] G. Ecker, A. Pich, and E. de Rafael, $K \rightarrow$ pi Lepton+Lepton-Decays in the Effective Chiral Lagrangian of the Standard Model, Nucl. Phys. B291 (1987) 692-719.

[59] G. D’Ambrosio, G. Ecker, G. Isidori, and J. Portoles, The Decays $K \longrightarrow$ pi $l+l$ - beyond leading order in the chiral expansion, JHEP 08 (1998) 004, [hep-ph/ 9808289 ].

[60] G. Isidori, G. Martinelli, and P. Turchetti, Rare kaon decays on the lattice, Phys. Lett. B633 (2006) 75-83, [hep-lat/0506026].

[61] RBC, UKQCD Collaboration, N. H. Christ, X. Feng, A. Portelli, and C. T. Sachrajda, Prospects for a lattice computation of rare kaon decay amplitudes: $K \rightarrow \pi \ell^{+} \ell^{-}$decays, Phys. Rev. D92 (2015), no. 9 094512, [1507.03094].

[62] N. H. Christ, X. Feng, A. Portelli, and C. T. Sachrajda, Prospects for a lattice computation of rare kaon decay amplitudes II $K \rightarrow \pi v \bar{v}$ decays, 1605.04442 .

[63] H. B. Meyer, Lattice QCD and the Timelike Pion Form Factor, Phys. Rev. Lett. 107 (2011) 072002, [1105.1892].

[64] X. Feng, S. Aoki, S. Hashimoto, and T. Kaneko, Timelike pion form factor in lattice QCD, Phys. Rev. D91 (2015), no. 5 054504, [1412.6319].

[65] C. Helmes, C. Jost, B. Knippschild, L. Liu, C. Urbach, M. Werner, and Z. Wang, The Rho Resonance from $N_{f}=2+1+1$ Twisted Mass Lattice QCD, in Proceedings, 33rd International Symposium on Lattice Field Theory (Lattice 2015), 2015. 1512.00282. 